\title{
STUDIES OF THE DYNAMIC STRUCTURE AND SPECTRA OF SOLAR X-RAY FLARES
}

S. W. KAHLER, A. S. KRIEGER, J. K. SILK, and R. W. SIMON, American Science and Engineering, Cambridge, Mass., U.S.A.

\author{
A. F. TIMOTHY \\ NASA Headquarters \\ and \\ G. VAIA NA \\ Center for Astrophysics, Harvard College Observatory - Smithsonian \\ Astrophysical Observatory, Cambridge, Mass., U.S.A.
}

Summary. Data from the AS \& E X-ray spectrographic telescope on Skylab were used for a preliminary study of two solar flares on 1973, August 9 and September 5. Photographic images taken through broad band filters during the early flare onsets and subsequent evolution of the flares were analyzed by microdensitometry and subsequent conversion to energy maps of the flare areas.

Three exposures of 4, 16, and 64 s taken of McMath plage region 12474 were used to study the preflare configuration of the August 9 flare. Although all three images were taken before the onset of the flare as determined by Solrad, microwave, and $\mathrm{H} \alpha$ reports, a significant X-ray flux enhancement is apparent in a bright coronal X-ray spot. The center of the flux increase coincides with the center of the X-ray spot and has a linear dimension first of $6 "-8^{\prime \prime}$ as determined from the 4 and $16 \mathrm{~s}$ exposures and later of $10^{\prime \prime}-12^{\prime \prime}$ as determined from the 16 and $64 \mathrm{~s}$ exposures.

The flare of September 5 occurred in one of three bright X-ray spots surrounding a large negative sunspot. Two frames taken after the onset of type III bursts but before the X-ray onset as seen in the Solrad data indicated a flux increase in a region with dimensions of about $8^{\prime \prime} \times 20^{\prime \prime}$. During the rising phase of the flare event the shape of the emitting region was changing with $\mathrm{X}$-ray fluxes increasing fastest in the northwest part of the flare where a small appendage to the main flare region appeared. A comparison of preflare and postflare images indicated that the X-ray spot in which the flare occurred nearly disappeared after the flare, but a second spot which did not flare showed enhanced intensity.

Future work will relate the positions of the flares and flare onsets to the pre-flare $\mathrm{X}$-ray structures, the magnetic fields, and the $\mathrm{H} \alpha$ features of the active regions. The photographic images will also be used to derive flare volumes and rates of change of the X-ray flux in various regions of the flares. 